\title{
On the generalized Ulam-Hyers-Rassias stability for quartic functional equation in modular spaces
}

\author{
Kittipong Wongkum ${ }^{\mathrm{a}, \mathrm{b}}$, Poom Kumam ${ }^{\mathrm{a}, \mathrm{b}, \mathrm{c}, *}$, Yeol Je Cho ${ }^{\mathrm{b}, \mathrm{d}}$, Phatiphat Thounthong ${ }^{\mathrm{e}, \mathrm{f}}$, Parin Chaipunya ${ }^{\mathrm{a}, \mathrm{b}}$ \\ ${ }^{a}$ KMUTT-Fixed Point Research Laboratory, Department of Mathematics, Room SCL 802 Fixed Point Laboratory, Science Laboratory \\ Building, Faculty of Science, King Mongkut's University of Technology Thonburi (KMUTT), 126 Pracha Uthit Road, Bang Mod, Thung \\ Khru, Bangkok 10140, Thailand. \\ ${ }^{b}$ KMUTT-Fixed Point Theory and Applications Research Group, Theoretical and Computational Science (TaCS) Center, Science \\ Laboratory Building, Faculty of Science, King Mongkut's University of Technology Thonburi (KMUTT), 126 Pracha Uthit Road, Bang \\ Mod, Thung Khru, Bangkok 10140, Thailand. \\ ${ }^{c}$ Department of Medical Research, China Medical University Hospital, China Medical University, Taichung 40402, Taiwan. \\ ${ }^{d}$ Department of Mathematics Education, Gyeongsang Natoinal University, Jinju 660-701, Korea. \\ ${ }^{e}$ Renewable Energy Research Centre, King Mongkuts University of Technology North Bangkok (KMUTNB), Wongsawang, Bangsue, \\ Bangkok 10800, Thailand. \\ ${ }^{f}$ Department of Teacher Training in Electrical Engineering, Faculty of Technical Education, King Mongkuts University of Technology \\ North Bangkok (KMUTNB), Wongsawang, Bangsue, Bangkok 10800, Thailand.
}

Communicated by S. Argyros

\begin{abstract}
In this paper, we prove the generalized UHR stability of a quartic functional equations $f(2 x+y)+f(2 x-y)=4 f(x+y)+$ $4 f(x-y)+24 f(x)-6 f(y)$ via the extensive studies of fixed point theory. Our results are obtained in the framework of modular spaces by the modular which is 1.s.c. and convex. (c)2017 All rights reserved.
\end{abstract}

Keywords: Quartic mapping, generalized UHR stability, modular space. 2010 MSC: 39B82, 47H10.

\section{Introduction}

The investigation of stability of functional equations began in 1940 by Ulam [10]. During a conference at Wisconsin University, Ulam posted the question asking about the stability of group homomorphisms as following:

Let $G_{1}$ be a group and let $G_{2}$ be a metric group with the metric $d(\cdot, \cdot)$. Given $\epsilon>0$, does there exist a $\delta>0$ such that if a function $f: G_{1} \rightarrow G_{2}$ satisfies the inequality $d(f(x y), f(x) f(y))<\delta$ for all $x, y \in G_{1}$, then there exists a homomorphism $g: G_{1} \rightarrow G_{2}$ with $d(f(x), g(x))<\epsilon$ for all $x \in G_{1}$ ?

\footnotetext{
*Corresponding author

Email addresses: kittipong.wong@mail.kmutt.ac.th (Kittipong Wongkum), poom.kum@kmutt.ac.th (Poom Kumam), yjcho@gnu.ac.kr, yjchomath@gmail.com (Yeol Je Cho), phtt@kmutnb.ac.th (Phatiphat Thounthong), parin.cha@mail.kmutt.ac.th (Parin Chaipunya)

doi:10.22436/jnsa.010.04.10
} 
That is, we are interested in the situation when the homomorphisms are stable, i.e., if a mapping is almost a homomorphism, then there exists an exact homomorphism close to it. In 1941, Hyers [4] gave a partial solution of Ulam's problem under the assumption that $G_{1}$ and $G_{2}$ are Banach spaces. This is the provenance of Hyers-Ulam stability of functional equation. In 1950, Aoki [1] generalized Hyers' theorem for approximately additive functions. In 1978, Rassias [8] generalized the theorem of Hyers to an unbounded case. The result of Rassias has had a lot of influences in the development of what we now call Ulam-Hyers-Rassias stability of functional equations. The stability problem of the functional equations has been investigated by many mathematicians and you can see in $[2,3]$.

The first author who treated the stability of the quartic equation was Rassias [9] for function $F: X \rightarrow Y$, where $X$ is a linear space and $Y$ is a Banach space. The stability problem of the quartic equation has been investigated by many mathematicians. For the example, Lee et al. [7] solved quartic functional equation and proved the stability of a quartic functional equation for function $f: X \rightarrow Y$, where $X, Y$ are real vector spaces. Lee and Chung [6] presented the general solution of a generalization of quartic functional equation in the class of functions between real vector spaces.

In this work, we introduce the following function equation in modular spaces

$$
f(2 x+y)+f(2 x-y)=4 f(x+y)+4 f(x-y)+24 f(x)-6 f(y),
$$

where a mapping in (1.1) is called a quartic functional equation and every solution of the quartic functional equation (in form: $f(x)=c x^{4}$ ) is said to be quartic function. We consider the case where $X$ is a linear space and $\mathrm{Y}$ is a $\rho$-complete modular space, whose the scalar fields are arbitrary. Our main results are obtained by using the fixed point method under the assumptions that the modular is lower semicontinuous (in brief 1.s.c.) and convex.

\section{Preliminaries}

In this section, we recall some basic definitions and properties of a modular space. In addition, we use $\mathbb{R}, \mathbb{C}$, and $\mathbb{N}$ to denote the set of all reals, complexes, and nonnegative integers, respectively.

Definition 2.1. Let $X$ be a vector space over a field $\mathbb{K}(\mathbb{R}$ or $\mathbb{C})$. A generalized functional $\rho: X \rightarrow[0, \infty]$ is called a modular if for arbitrary $x, y \in X$,

(m1) $\rho(x)=0$ if and only if $x=0$;

(m2) $\rho(\alpha x)=\rho(x)$ for every scalar $\alpha$ with $|\alpha|=1$;

(m3) $\rho(z) \leqslant \rho(x)+\rho(y)$ whenever $z$ is a convex combination of $x$ and $y$.

The corresponding modular space, denoted by $X_{\rho}$, is then defined by

$$
X_{\rho}:=\{x \in X: \rho(\lambda x) \longrightarrow 0 \text { as } \lambda \longrightarrow 0\} .
$$

Remark 2.2. Note that for a fixed $x \in X_{\rho}$, the valuation $\gamma \in \mathbb{K} \mapsto \rho(\gamma x)$ is increasing.

Continuity or convexity in a modular is not necessary. However, it often occurs that some weaker form of them are assumed.

Remark 2.3. In case a modular $\rho$ is convex, one has $\rho(x) \leqslant \delta \rho\left(\frac{1}{\delta} x\right)$ for all $x \in X_{\rho}$, provided that $0<\delta \leqslant 1$.

Definition 2.4. Let $X_{\rho}$ be a modular space and $\left\{x_{n}\right\}$ be a sequence in $X_{\rho}$. Then,

(i) $\left\{x_{n}\right\}$ is $\rho$-convergent to a point $x \in X_{\rho}$ and write $x_{n} \stackrel{\rho}{\rightarrow} x$ if $\rho\left(x_{n}-x\right) \rightarrow 0$ as $n \rightarrow \infty$.

(ii) $\left\{x_{n}\right\}$ is called $\rho$-Cauchy if for any $\epsilon>0$, we have $\rho\left(x_{n}-x_{m}\right)<\epsilon$ for sufficiently large $m, n \in \mathbb{N}$.

(iii) A subset $K \subset X_{\rho}$ is called $\rho$-complete if any $\rho$-Cauchy sequence is $\rho$-convergent. 
In modular spaces, the convergence of a sequence $\left\{x_{n}\right\}$ to $x$ does not imply that $\left\{c x_{n}\right\}$ converges to $c x$, where $c$ is chosen from the corresponding scalar field. So, it is necessary to add some conditions. Such preferences are referred to mostly under the term related to the $\Delta_{2}$-conditions.

A modular $\rho$ is said to satisfy the $\Delta_{2}$-condition if there exists $\kappa \geqslant 2$ such that $\rho(2 x) \leqslant \kappa \rho(x)$ for all $x \in X_{\rho}$. Some authors varied the notion so that only $k>0$ is required and called it the $\Delta_{2}$-type condition. In fact, one may see that these two notions coincide. There are still a number of equivalent notions related to the $\Delta_{2}$-conditions.

Remark 2.5. The averaged sequence $\left\{\frac{1}{2^{k}} \sum_{i=1}^{2 k} \chi_{n}^{i}\right\} \rho$-converges to $\frac{1}{2^{k}} \sum_{i=1}^{2 k} \chi^{i}$, where we suppose that $\left\{x_{n}^{1}\right\}$, $\left\{x_{\mathrm{n}}^{2}\right\}, \cdots,\left\{x_{\mathrm{n}}^{2 \mathrm{k}}\right\}$, for some $k \in \mathbb{N}$, are sequences in $X_{\rho}$ in which they $\rho$-converge to the points $\chi^{1}, x^{2}, \ldots, x^{2 \mathrm{k}} \in$ $X_{\rho}$, respectively.

In [5], Khamsi proved a series of fixed point theorems in modular spaces. His results exploit one unifying hypothesis in which the boundedness of an orbit is assumed. Moreover, if it happens to have more than one fixed points, say $x$ and $y$, then it must be the case that $\rho(x-y)=\infty$.

Definition 2.6. Given a modular space $X_{\rho}$, a nonempty subset $C \subset X_{\rho}$, and a mapping $T: C \rightarrow C$, the orbit of $\mathrm{T}$ around a point $\mathrm{x} \in \mathrm{C}$ is the set

$$
\mathcal{O}(x):=\left\{x, T x, T^{2} x, \cdots\right\}
$$

The quantity $\delta_{\rho}(x):=\sup \{\rho(u-v): u, v \in \mathcal{O}(x)\}$ is then associated and is called the orbital diameter of $\mathrm{T}$ at $x$. In particular, if $\delta_{\rho}(x)<\infty$, we say that T has a bounded orbit at $x$.

Lemma 2.7 ([5]). Let $\mathrm{X}_{\rho}$ be a modular space whose the induced modular is l.s.c. and $\mathrm{C} \subset \mathrm{X}_{\rho}$ be a $\rho$-complete subset. If $\mathrm{T}: \mathrm{C} \rightarrow \mathrm{C}$ is a $\rho$-contraction, i.e., there is a constant $\mathrm{k} \in[0,1)$ such that

$$
\rho(T x-T y) \leqslant k \rho(x-y), \quad \forall x, y \in C,
$$

and $\mathrm{T}$ has a bounded orbit at a point $\mathrm{x}_{0} \in \mathrm{C}$, then the sequence $\left\{\mathrm{T}^{\mathrm{n}} \mathrm{x}_{0}\right\}$ is $\rho$-convergent to a point $w \in \mathrm{C}$.

\section{Main results}

This section describes details stability of quartic functional equation in modular spaces. The prove method requires no $\Delta_{2}$-conditions which is different from other researches.

Definition 3.1. For a constant $L \geqslant 0$ and a linear space $V$, we define $\Phi_{V, L}$ to be the collection of all nonnegative real-valued functions $\psi$ defined on $V$ with the following properties for all $x, y \in V$ :

$$
\left\{\begin{array}{l}
\lim _{n \rightarrow \infty} \psi\left(2^{n} x, y\right) / 16^{n}=0, \\
\psi(2 x, 0) \leqslant 16 \operatorname{L} \psi(x, 0) .
\end{array}\right.
$$

Theorem 3.2. Let $\mathrm{V}$ be linear space, $\mathrm{X}_{\rho}$ be a $\rho$-complete modular space where $\rho$ is l.s.c. and convex, and $\mathrm{f}: \mathrm{V} \rightarrow \mathrm{X}_{\rho}$ be a mapping with $\mathrm{f}(0)=0$. Suppose that for each $\mathrm{x}, \mathrm{y} \in \mathrm{V}$, the following dominating condition holds:

$$
\rho(f(2 x+y)+f(2 x-y)-4 f(x+y)-4 f(x-y)-24 f(x)+6 f(y)) \leqslant \phi(x, y),
$$

where $\phi \in \Phi_{\mathrm{V}, \mathrm{L}}$ with $0<\mathrm{L}<1$. Then, there exists a quartic mapping $w: \mathrm{V} \rightarrow \mathrm{X}_{\rho}$ such that

$$
\rho(w(x)-f(x)) \leqslant \frac{1}{16(1-L)} \phi(x, 0)
$$

for all $\mathrm{x} \in \mathrm{V}$. Equivalently, the quartic mapping is generalized UHR stable.

To prove this stability result, we need the following lemma. 
Lemma 3.3. Suppose that all assumptions of Theorem 3.2 hold. Then, the following statements hold:

(S1) The set $\mathcal{M}:=\left\{g: V \rightarrow X_{\rho}: g(0)=0\right\}$ is a linear space.

(S2) A generalized function $\tilde{\rho}$ defined for each $\mathrm{g} \in \mathcal{M}$ by

$$
\tilde{\rho}(g):=\inf \{c>0: \rho(g(x)) \leqslant c \phi(x, 0), \forall x \in V\}
$$

is a convex modular on $\mathcal{M}$.

(S3) The corresponding modular space $\mathcal{M}_{\tilde{\rho}}$ is the whole space $\mathcal{M}$ and is $\tilde{\rho}$-complete.

(S4) $\tilde{\rho}$ is l.s.c.

Proof. (S1) is trivial.

To prove (S2) it is also easy to verify that $\tilde{\rho}$ satisfies the axioms (m1) and (m2) of a modular. Next we shall show that $\tilde{\rho}$ is convex, and hence (m3) is satisfied. Let $\epsilon>0$ be given. Then there exist $c_{1}>0$ and $\mathrm{c}_{2}>0$ such that

$$
\tilde{\rho}(g) \leqslant c_{1} \leqslant \tilde{\rho}(g)+\epsilon \text { and } \tilde{\rho}(h) \leqslant c_{2} \leqslant \tilde{\rho}(h)+\epsilon .
$$

Consecutively, we have

$$
\rho(g(x)) \leqslant c_{1} \phi(x, 0) \text { and } \rho(h(x)) \leqslant c_{2} \phi(x, 0) .
$$

Thus, if $\alpha+\beta=1$ and $\alpha, \beta \geqslant 0$, we get

$$
\rho(\alpha g(x)+\beta h(x)) \leqslant \alpha \rho(g(x))+\beta \rho(h(x)) \leqslant\left(\alpha c_{1}+\beta c_{2}\right) \phi(x, 0),
$$

so that

$$
\tilde{\rho}(\alpha g+\beta h) \leqslant \alpha \tilde{\rho}(g)+\beta \tilde{\rho}(h)+(\alpha+\beta) \epsilon .
$$

Hence, we have

$$
\tilde{\rho}(\alpha g+\beta h) \leqslant \alpha \tilde{\rho}(g)+\beta \tilde{\rho}(h) .
$$

This concludes that $\tilde{\rho}$ is a convex modular on $\mathcal{M}$.

For (S3), the fact that the corresponding modular space $\mathcal{M}_{\tilde{\rho}}$ is the whole space $\mathcal{M}$ is trivial, so we only show that $\mathcal{M}_{\tilde{\rho}}$ is $\tilde{\rho}$-complete. Let $\left\{g_{n}\right\}$ be a $\tilde{\rho}$-Cauchy sequence in $\mathcal{M}_{\tilde{\rho}}$ and let $\epsilon>0$ be given. There exists a positive integer $n_{0} \in \mathbb{N}$ such that $\tilde{\rho}\left(g_{n}-g_{m}\right)<\epsilon$ for all $n, m \geqslant n_{0}$. By the definition, we may see that

$$
\rho\left(g_{n}(x)-g_{m}(x)\right) \leqslant \epsilon \phi(x, 0)
$$

for all $x \in V$ and $n, m \geqslant n_{0}$. Thus, at each fixed $x \in V$, the sequence $\left\{g_{n}(x)\right\}$ is a $\rho$-Cauchy sequence. Since $X_{\rho}$ is $\rho$-complete, so $\left\{g_{n}(x)\right\}$ is $\rho$-convergent in $X_{\rho}$ for each $x \in V$. Hence, we can define a function $g: V \rightarrow X_{\rho}$ by

$$
g(x):=\lim _{n \rightarrow \infty} g_{n}(x)
$$

for any $x \in$ V. Since $\rho$ is 1.s.c., it follows from (3.2) that

$$
\rho\left(g_{\mathfrak{n}}(x)-g(x)\right) \leqslant \liminf _{m \rightarrow \infty} \rho\left(g_{\mathfrak{n}}(x)-g_{m}(x)\right) \leqslant \epsilon \phi(x, 0),
$$

provided that $n \geqslant n_{0}$. Thus, $\left\{g_{n}\right\} \tilde{\rho}$-converges, so that $\mathcal{M}_{\tilde{\rho}}$ is $\tilde{\rho}$-complete.

For (S4), suppose that $\left\{g_{n}\right\}$ is a sequence in $\mathcal{M}_{\tilde{\rho}}$ which is $\tilde{\rho}$-convergent to an element $g \in \mathcal{M}_{\tilde{\rho}}$. Let $\epsilon>0$ be given. For each $n \in \mathbb{N}$, let $c_{n}$ be a constant such that

$$
\tilde{\rho}\left(g_{n}\right) \leqslant c_{n} \leqslant \tilde{\rho}\left(g_{n}\right)+\epsilon .
$$

Again, we have

$$
\rho\left(g_{n}(x)\right) \leqslant c_{n} \phi(x, 0), \quad \forall x \in V
$$


Now, observe from the lower semicontinuity of $\rho$ that

$$
\rho(g(x)) \leqslant \liminf _{n \rightarrow \infty} \rho\left(g_{n}(x)\right) \leqslant \liminf _{n \rightarrow \infty} c_{n} \phi(x, 0) \leqslant\left[\liminf _{n \rightarrow \infty} \tilde{\rho}\left(g_{n}\right)+\epsilon\right] \phi(x, 0) .
$$

Thus, we have

$$
\tilde{\rho}(g) \leqslant \liminf _{n \rightarrow \infty} \tilde{\rho}\left(g_{n}\right)+\epsilon .
$$

Since $\epsilon>0$ is arbitrary, we can finally conclude that $\tilde{\rho}$ is l.s.c.

Next, we show that a self-mapping $\mathrm{T}: \mathcal{M}_{\tilde{\rho}} \rightarrow \mathcal{M}_{\tilde{\rho}}$ defined by

$$
\operatorname{Tg}(x):=\frac{1}{16} g(2 x), \quad \forall g \in \mathcal{M}_{\tilde{\rho}}, \forall x \in V
$$

has some fixed points according to Lemma 2.7.

Remark 3.4. It is useful to notice that $\mathrm{T}$ is a linear mapping.

Lemma 3.5. Suppose that all assumptions of Theorem 3.2 hold and $\mathrm{T}$ is defined as in (3.3). Then, $\mathrm{T}$ has a fixed point.

Proof. We first show that $\mathrm{T}$ is a $\tilde{\rho}$-contraction. Let $x \in \mathrm{V}, \mathrm{g}, \mathrm{h} \in \mathcal{M}_{\tilde{\rho}}$ and $\mathrm{c} \in[0, \infty]$ be an arbitrary constant with $\tilde{\rho}(g-h) \leqslant c$. Observe that we have

$$
\rho(g(x)-h(x)) \leqslant c \phi(x, 0),
$$

so that

$$
\rho\left(\frac{g(2 x)}{16}-\frac{h(2 x)}{16}\right) \leqslant \frac{1}{16} \rho(g(2 x)-h(2 x)) \leqslant \frac{1}{16} c \phi(2 x, 0) \leqslant \operatorname{Lc} \phi(x, 0) .
$$

Therefore, we have $\tilde{\rho}(\mathrm{Tg}-\mathrm{Th}) \leqslant \mathrm{L} \tilde{\rho}(\mathrm{g}-\mathrm{h})$. Since $g, h \in \mathcal{M}_{\tilde{\rho}}$ are arbitrary, $\mathrm{T}$ is a $\tilde{\rho}$-strict contraction w.r.t. L.

Next, we show that $T$ has a bounded orbit at $f$, where $f$ is taken from the assumption. Let $x \in V$ be arbitrary and set $y=0$ in (3.1), we get

$$
\rho(2 f(2 x)-32 f(x)) \leqslant \phi(x, 0), \quad \forall x \in V .
$$

Set $x=2 x$ in (3.4), we get

$$
\rho(2 f(4 x)-32 f(2 x)) \leqslant \phi(2 x, 0), \quad \forall x \in V .
$$

Since $\rho$ is convex, we obtain

$$
\begin{aligned}
\rho(f(2 x)-16 f(x)) & =\rho\left(\frac{2}{2} \cdot f(2 x)-\frac{2}{2} \cdot 16 f(x)\right) \\
& =\rho\left(\frac{1}{2}(2 f(2 x)-32 f(x))+\frac{1}{2} \cdot 0\right) \\
& \leqslant \frac{1}{2} \rho(2 f(2 x)-32 f(x)) \leqslant \frac{1}{2} \phi(x, 0), \quad \forall x \in V .
\end{aligned}
$$

Moreover,

$$
\begin{aligned}
\rho\left(\frac{f\left(2^{2} x\right)}{16}-16 f(x)\right) & =\rho\left(\frac{2}{2} \cdot \frac{f\left(2^{2} x\right)}{16}-\frac{2}{2} f(2 x)+\frac{2}{2} f(2 x)-\frac{2}{2} \cdot 16 f(x)\right) \\
& =\rho\left[\frac{1}{2}\left(\frac{2 f\left(2^{2} x\right)}{16}-2 f(2 x)\right)+\frac{1}{2}(2 f(2 x)-32 f(x))\right]
\end{aligned}
$$




$$
\begin{aligned}
& \leqslant \frac{1}{2} \rho\left(\frac{2 f\left(2^{2} x\right)}{16}-2 f(2 x)\right)+\frac{1}{2} \rho(2 f(2 x)-32 f(x)) \\
& =\frac{1}{2} \rho\left(\frac{1}{16}\left(2 f\left(2^{2} x\right)-32 f(2 x)\right)\right)+\frac{1}{2} \rho(2 f(2 x)-32 f(x)) \\
& \leqslant \frac{1}{2} \cdot \frac{1}{16} \rho\left(2 f\left(2^{2} x\right)-32 f(2 x)\right)+\frac{1}{2} \rho(2 f(2 x)-32 f(x)) \\
& \leqslant \frac{1}{2}\left(\frac{1}{16} \phi(2 x, 0)+\phi(x, 0)\right), \quad \forall x \in V .
\end{aligned}
$$

By induction, we may deduce for all $j \in N$ that

$$
\rho\left(\frac{f\left(2^{j} x\right)}{16^{j-1}}-16 f(x)\right) \leqslant \frac{1}{2} \Sigma_{j=1}^{j} \frac{1}{16^{j-1}} \phi\left(2^{j-1} x, 0\right), \quad \forall x \in V .
$$

Moreover, we get

$$
\begin{aligned}
\rho\left(\frac{f\left(2^{j} x\right)}{16^{j-1}}-16 f(x)\right) & \leqslant \frac{1}{2} \sum_{j=1}^{j} \frac{1}{16^{j-1}} \phi\left(2^{j-1} x, 0\right), \quad \forall x \in V . \\
& =\frac{1}{2}\left[\frac{1}{16^{j-1}} \phi\left(2^{j-1} x, 0\right)+\frac{1}{16^{j-2}} \phi\left(2^{j-2} x, 0\right)+\cdots+\frac{1}{16^{j-j}} \phi\left(2^{j-j} x, 0\right)\right] \\
& =\frac{1}{2}\left[\phi(x, 0)+\frac{1}{16} \phi(2 x, 0)+\cdots+\frac{1}{16^{j-1}} \phi\left(2^{j-1} x, 0\right)\right] \\
& \leqslant \frac{1}{2}\left[\phi(x, 0)+\phi(x, 0) \cdot \mathrm{L}+\phi(x, 0) \cdot \mathrm{L}^{2}+\ldots+\phi(x, 0) \cdot \mathrm{L}^{j-1}\right] \\
& =\frac{1}{2} \phi(x, 0)\left[1+\mathrm{L}+\mathrm{L}^{2}+\cdots+\mathrm{L}^{j-1}\right] \\
& \leqslant \frac{1}{2} \phi(x, 0)\left[1+\mathrm{L}+\mathrm{L}^{2}+\cdots\right] \\
& =\frac{1}{2(1-\mathrm{L})} \phi(x, 0), \quad \forall x \in \mathrm{V} .
\end{aligned}
$$

Now, for each $m, n \in \mathbb{N}$, we have

$$
\begin{aligned}
\rho\left(\frac{f\left(2^{n} x\right)}{16^{n}}-\frac{f\left(2^{m} x\right)}{16^{m}}\right) & =\rho\left(\frac{2}{2} \cdot \frac{f\left(2^{n} x\right)}{16^{n}}-\frac{2}{2} \cdot f(x)+\frac{2}{2} \cdot f(x)-\frac{2}{2} \cdot \frac{f\left(2^{m} x\right)}{16^{m}}\right) \\
& =\rho\left(\frac{1}{2}\left(\frac{2 f\left(2^{n} x\right)}{16^{n}}-2 f(x)\right)+\frac{1}{2}\left(2 f(x)-\frac{2 f\left(2^{m} x\right)}{16^{m}}\right)\right) \\
& \leqslant \frac{1}{2}\left[\rho\left(\frac{2 f\left(2^{n} x\right)}{16^{n}}-2 f(x)\right)+\rho\left(2 f(x)-\frac{2 f\left(2^{m} x\right)}{16^{m}}\right)\right] \\
& \leqslant \frac{1}{2}\left[\frac{1}{8} \rho\left(\frac{f\left(2^{n} x\right)}{16^{n-1}}-16 f(x)\right)+\frac{1}{8} \rho\left(16 f(x)-\frac{f\left(2^{m} x\right)}{16^{m}-1}\right)\right] \\
& \leqslant \frac{1}{2}\left[\frac{1}{8} \cdot \frac{1}{2(1-L)} \phi(x, 0)+\frac{1}{8} \cdot \frac{1}{2(1-L)} \phi(x, 0)\right] \\
& =\frac{1}{16(1-L)} \phi(x, 0), \quad \forall x \in V .
\end{aligned}
$$

By the definition of $\tilde{\rho}$, we conclude that

$$
\tilde{\rho}\left(T^{n_{f}}-T^{m_{f}}\right) \leqslant \frac{1}{16(1-L)}<\infty,
$$


which implies the boundedness of an orbit of $T$ at $f$. According to Lemma 2.7, the sequence $\left\{T^{n} f\right\} \rho$ converges to some element, say $w \in \mathcal{M}_{\tilde{\rho}}$. Now, by the $\tilde{\rho}$-contractivity of $T$, one has

$$
\tilde{\rho}\left(T w-T^{n+1} f\right) \leqslant L \tilde{\rho}\left(w-T^{n} f\right) .
$$

Passing $\mathrm{n}$ towards $\infty$ and applying the lower semicontinuity of $\tilde{\rho}$, we obtain that

$$
\tilde{\rho}(T w-w) \leqslant \liminf _{n \rightarrow \infty} \tilde{\rho}\left(T w-T^{n+1} f\right) \leqslant \liminf _{n \rightarrow \infty} L \tilde{\rho}\left(w-T^{n} f\right)=0 .
$$

Therefore, $w$ is a fixed point of $\mathrm{T}$.

Now, with the two lemmas above, we can finally give a simple proof to our main stability result, namely Theorem 3.2.

Proof of Theorem 3.2. Since $2^{n} x$ is in $V$ provided that $x \in V$, we deduce from (3.1) that

$$
\begin{aligned}
& \rho\left(f\left(2^{n}(2 x+y)\right)+f\left(2^{n}(2 x-y)\right)-4 f\left(2^{n}(x+y)\right)-4 f\left(2^{n}(x-y)\right)-24 f\left(2^{n}(x)\right)+6 f\left(2^{n}(y)\right)\right) \\
& \leqslant \phi\left(2^{n} x, 2^{n} y\right), \quad \forall x, y \in V .
\end{aligned}
$$

Furthermore, we have

$$
\begin{aligned}
& \rho\left(\frac{f\left(2^{n}(2 x+y)\right)}{16^{n}}+\frac{f\left(2^{n}(2 x-y)\right)}{16^{n}}-\frac{4 f\left(2^{n}(x+y)\right)}{16^{n}}-\frac{4 f\left(2^{n}(x-y)\right)}{16^{n}}-\frac{24 f\left(2^{n}(x)\right)}{16^{n}}+\frac{6 f\left(2^{n}(y)\right)}{16^{n}}\right) \\
& \quad \leqslant \frac{1}{16^{n}} \rho\left(f\left(2^{n}(2 x+y)\right)+f\left(2^{n}(2 x-y)\right)-4 f\left(2^{n}(x+y)\right)-4 f\left(2^{n}(x-y)\right)-24 f\left(2^{n}(x)\right)+6 f\left(2^{n}(y)\right)\right) \\
& \quad \leqslant \frac{\phi\left(2^{n} x, 2^{n} y\right)}{16^{n}}, \quad \forall x, y \in V .
\end{aligned}
$$

As from Lemma 3.5 and Remark 2.5, letting $n \longrightarrow \infty$ and applying the lower semicontinuity of $\rho$, we deduce that

$$
w(2 x+y)+w(2 x-y)=4 w(x+y)+4 w(x-y)+24 w(x)-6 w(y), \quad \forall x, y \in V .
$$

That is, $w$ is a quadratic mapping. Since every quadratic map is a fixed point of T, Lemma 3.5 also guarantees the uniqueness of $w$. On the other hand, it follows from inequality (3.5) that

$$
\tilde{\rho}(w-f) \leqslant \frac{1}{16(1-L)} .
$$

Corollary 3.6. Let $\mathrm{V}$ be a linear space, $\mathrm{X}_{\rho}$ be a $\rho$-complete modular space where $\rho$ is l.s.c. and convex, and $\mathrm{f}: \mathrm{V} \rightarrow \mathrm{X}_{\rho}$ be a mapping with $\mathrm{f}(0)=0$. If there exists a constant $\delta>0$ such that

$$
\rho(f(2 x+y)+f(2 x-y)-4 f(x+y)-4 f(x-y)-24 f(x)+6 f(y)) \leqslant \delta, \quad \forall x, y \in V,
$$

then there exists a unique quadratic mapping $w: V \rightarrow X_{\rho}$ such that

$$
\rho(w(x)-f(x)) \leqslant \frac{\delta}{15}, \quad \forall x \in V .
$$

For the next two corollaries, we shall consider the case where $\rho$ is actually a norm.

Corollary 3.7. Let $\mathrm{V}$ be a linear space, $(\mathrm{X},\|\cdot\|)$ be a Banach space, and $\mathrm{f}: \mathrm{V} \rightarrow \mathrm{X}$ be a mapping with $\mathrm{f}(0)=0$. Suppose that for each $\mathrm{x}, \mathrm{y} \in \mathrm{V}$, there holds the inequality

$$
\|f(2 x+y)+f(2 x-y)-4 f(x+y)-4 f(x-y)-24 f(x)+6 f(y)\| \leqslant \phi(x, y),
$$


where $\phi \in \Phi_{\mathrm{V}, \mathrm{L}}$ with $0<\mathrm{L}<1$. Then, there exists a unique quartic mapping $w: \mathrm{V} \rightarrow \mathrm{X}$ such that

$$
\|w(x)-f(x)\| \leqslant \frac{1}{16(1-L)} \phi(x, 0), \quad \forall x \in V .
$$

Corollary 3.8. Let $\mathrm{V}$ be a linear space, $(\mathrm{X},\|\cdot\|)$ be a Banach space, and $\mathrm{f}: \mathrm{V} \rightarrow \mathrm{X}$ be a mapping with $\mathrm{f}(0)=0$. Suppose there exists a constant $\delta>0$ such that

$$
\|f(2 x+y)+f(2 x-y)-4 f(x+y)-4 f(x-y)-24 f(x)+6 f(y)\| \leqslant \delta, \quad \forall x, y \in V,
$$

then there exists a unique quartic mapping $w: \mathrm{V} \rightarrow \mathrm{X}$ such that

$$
\|w(x)-f(x)\| \leqslant \frac{\delta}{15}, \quad \forall x \in V .
$$

\section{Concluding remarks}

Our results guarantee the stability of quartic mappings, whose codomain is equipped with a convex and l.s.c. modular, in both generalized and original senses. Our proofs contain different techniques to avoid the usage of $\Delta_{2}$-conditions.

\section{Acknowledgment}

The authors are gratefully thankful for the referee's valuable comments, which significantly improve materials in this paper. This project was supported by the Theoretical and Computational Science (TaCS) Center under Computational and Applied Science for Smart Innovation Cluster (CLASSIC), Faculty of Science, KMUTT. Also, this research work was financially supported by the King Mongkut's University of Technology North Bangkok Contract No. KMUTNB-60-ART-086.

\section{References}

[1] T. Aoki, On the stability of the linear transformation in Banach spaces, J. Math. Soc. Japan, 2 (1950), 64-66. 1

[2] Y. J. Cho, C.-K. Park, T. M. Rassias, R. Saadati, Stability of functional equations in Banach algebras, Springer, Cham, (2015). 1

[3] Y. J. Cho, T. M. Rassias, R. Saadati, Stability of functional equations in random normed spaces, Springer Optimization and Its Applications, Springer, New York, (2013). 1

[4] D. H. Hyers, On the stability of the linear functional equation, Proc. Nat. Acad. Sci. U. S. A., 27 (1941), 222-224. 1

[5] M. A. Khamsi, Quasicontraction mappings in modular spaces without $\Delta_{2}$-condition, Fixed Point Theory Appl., 2008 (2008), 6 pages. 2, 2.7

[6] Y.-S. Lee, S.-Y. Chung, Stability of quartic functional equations in the spaces of generalized functions, Adv. Difference Equ., 2009 (2009), 16 pages. 1

[7] S. H. Lee, S. M. Im, I. S. Hwang, Quartic functional equations, J. Math. Anal. Appl., 307 (2005), 387-394. 1

[8] T. M. Rassias, On the stability of the linear mapping in Banach spaces, Proc. Amer. Math. Soc., 72 (1978), 297-300. 1

[9] J. M. Rassias, Solution of the Ulam stability problem for quartic mappings, Glas. Mat. Ser. III, 34(54) (1999), 243-252. 1

[10] S. M. Ulam, Problems in modern mathematics, Science Editions John Wiley \& Sons, Inc., New York, (1964). 1 